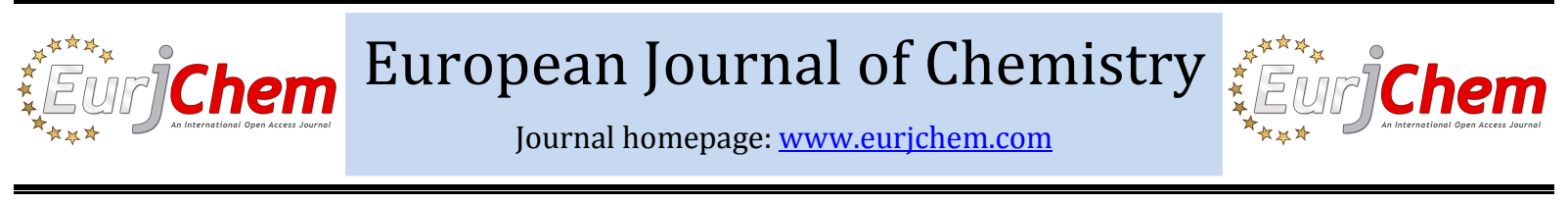

\title{
A study of coupling reaction to synthesize diphenylmethane derivatives
}

\author{
Huma Aslam Bhatti a,b,*, Qurat-Ul-Ain Zaheer a, Memoona Khatoon a, \\ Mark Edward Light $b$, and Abdul Hameed a
}

a Husein Ebrahim Jamal Research Institute of Chemistry, International Center for Chemical and Biological Sciences, University of Karachi, Karachi, 75270, Pakistan b School of Chemistry, University of Southampton, Highfield, Southampton, SO17 1BJ, England

*Corresponding author at: Husein Ebrahim Jamal Research Institute of Chemistry, International Center for Chemical and Biological Sciences, University of Karachi, Karachi, 75270, Pakistan.

Tel.: +92.111.222292. Fax: +92.213.4819018. E-mail address: huma aslam31@hotmail.com (H.A. Bhatti).

\section{ARTICLE INFORMATION}

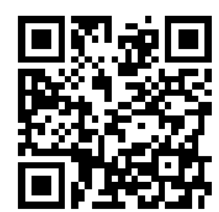

DOI: 10.5155/eurjchem.5.3.513-516.1086

Received: 28 April 2014

Received in revised form: 26 May 2014

Accepted: 01 June 2014

Online: 30 September 2014

\section{KEYWORDS}

Benzyl alcohol

Thionyl chloride

Coupling reaction

Halogenating agents

Phosphorus tribromide

Diphenylmethane derivative

\section{Introduction}

Diphenylmethane scaffold is found in various compounds of commercial importance in pharmaceutics [1] and fine chemical industries [2,3]. These compounds serve as important intermediates in the synthesis of new drug candidates $[4,5]$, adhesives and epoxy resins [6,7], preserves in perfumes and as solvent in pressure sensitive reaction [8]. The diphenyl methane derivatives are also added to improve the thermal stability of polyesters [9] and lubricating properties of jet fuels [10]. However, there are some diphenylmethane derivatives, which have been isolated from natural sources and possess significant bioactivities. A class of brominated diphenyl methane derivatives $\mathbf{1}$ which has been isolated from green alga Avrainvillea nigrlcans and possessed antibiotic activity against several human pathogens [11] (Figure 1). While, recently a new diphenylmethane derivative $\mathbf{2}$ has been isolated from the bioactive mixture of Periploca sepium which, exhibited activity against autoimmune diseases, especially for the treatment of rheumatoid in traditional Chinese medicines [12] (Figure 1).

The diverse applications of diphenylmethane derivatives make them interesting target for synthetic chemists. In literature, usually Friedel-Craft reactions that including acylation or alkylation were employed to prepare diphenylmethane derivatives.

\section{ABSTRACT}

The class of compounds having diphenylmethane framework occupy a distinct place in natural which, two benzyl alcohol molecules were coupled in the presence of different halogenating agents $\left(\mathrm{SOCl}_{2}, \mathrm{PBr}_{3}\right.$ and $\left.\mathrm{MeSO}_{2} \mathrm{Cl}\right)$ to afford the desired diphenylmethane derivative, bis(2,4ring. The resultant compound, bis(2,4-bis(benzyloxy)-5-methoxyphenyl)methane, was obtained in excellent yield (83-85\%) and completely characterize with different spectroscopic techniques. 
Table 1. Coupling reaction attempts with mono-substituted benzyl alcohol.

$\begin{array}{lllllll}\text { Entry } & \text { Reaction conditions } & \text { Diphenylmethane derivative } & \text { (6) } & \text { No product }\end{array}$

Also, both of the Friedel-Crafts reaction necessarily required Lewis acids such as $\mathrm{AlCl}_{3}, \mathrm{FeCl}_{3}$, or $\mathrm{ZnCl}$, as precatalyst. These acid catalysts offer toxicity and various environmental hazardous effects $[15,16]$. So, in the loop of diphenyl methane analogues synthesis, we wish to report a unique cross-coupling approach to synthesize the diphenyl methane derivatives from benzyl alcohol derivatives.

\section{Experimental}

\subsection{Materials}

All the benzyl chlorides, halogenating agents and solvents were purchased from different available commercial sources. Silica gel column chromatography was performed with silica gel 60 as the stationary phase with different analytical grade solvents i.e. EtOAc, hexane, petroleum ether. Chromatograms were visualized by UV at 254 and $365 \mathrm{~nm}$.

\subsection{Instrumentation}

NMR spectra were obtained on Advance Bruker AM 300 and $400 \mathrm{MHz}$. Single crystal X-ray diffraction data: Diffractometer: Nonius KappaCCD area detector ( $\phi$ scans and $\omega$ scans to fill asymmetric unit). Cell determination: DirAx [17], Data collection: COLLECT data collection software [18]. Data reduction and cell refinement: Denzo [19]. Absorption correction: Sheldrick, G. M. SADABS-Bruker Nonius area detector scaling and absorption correction-V2.10 Structure solution: SHELXS97 [20]. Structure refinement: SHELXL97 (G. M. Sheldrick (1997), University of Göttingen, Germany). Graphics: Cameron-A Molecular Graphics Package [21]. Special details: All hydrogen atoms were placed in idealized positions and refined using a riding model.

\subsection{Synthesis of diphenylmethane derivative, bis $(2,4-$ bis(benzyloxy)-5-methoxyphenyl)methane (12)}

Method 1: The solution of thionylchloride $\left(\mathrm{SOCl}_{2}\right)(0.01 \mathrm{~mL}$, $0.21 \mathrm{mmole})$ in $\mathrm{CH}_{2} \mathrm{Cl}_{2}$ was added dropwise to the cold $\left(0^{\circ} \mathrm{C}\right)$ stirring solution of 2,4-bis(benzyloxy)-5-methoxyphenyl) methanol 11 (100 mg, 0.28 mmoles) in $\mathrm{CH}_{2} \mathrm{Cl}_{2}$. The resulting mixture was stirred for $45 \mathrm{~min}$ at $0{ }^{\circ} \mathrm{C}$, at which the solution was poured into ice water and extracted with dichloromethane $\left(\mathrm{CH}_{2} \mathrm{Cl}_{2}\right)$. All the separated organic layers were combined, dried over $\mathrm{MgSO}_{4}$ and then subsequently evaporated dried the crude product by column chromatography to get the product $\mathbf{1 2}$ in 85 $\%$ yield. With phosphoroustribromide $\left(\mathrm{PBr}_{3}\right)$, same protocol was used to afford the diphenylmethane derivative 12 in 83\% yield.

Method 2: Methane sulphonyl chloride $(4.9 \mathrm{~mL}, 50.4$ mmols) was added dropwise to a solution of 2,4-bis(benzy loxy)-5-methoxyphenyl)methanol $\mathbf{1 1}$ (3.6 g, $12.6 \mathrm{mmoles}$ ) and $\mathrm{Et}_{3} \mathrm{~N}(7 \mathrm{~mL}, 50.4 \mathrm{mmol})$ in THF $(50 \mathrm{~mL})$ at $0{ }^{\circ} \mathrm{C}$. The resulting mixture was then stirred overnight at room temperature. The reaction was quenched with water and extracted with the $\mathrm{Et}_{2} \mathrm{O}$ and washed with brine, purified by column chromatography to get pure product $12(3.9 \mathrm{~g})$ in $83 \%$ yield. The structures of resultant compound $\mathbf{1 2}$ were confirmed with NMR spectroscopy, mass spectrometry and X-ray crystallography.

Bis(2,4-bis(benzyloxy)-5-methoxyphenyl)methane (12): Color: White crystals. ${ }^{1} \mathrm{H}$ NMR $\left(300 \mathrm{MHz}, \mathrm{CHCl}_{3}, \delta\right.$, ppm): 7.49 $7.10(\mathrm{~m}, 20 \mathrm{H}, \mathrm{ArH}), 6.52$ (s, 2H, ArH), 6.50 (s, 2H, ArH), 5.02 (s, $\left.4 \mathrm{H}, 2\left(\mathrm{CH}_{2}\right)\right), 4.80\left(\mathrm{~s}, 4 \mathrm{H}, 2\left(\mathrm{CH}_{2}\right)\right), 3.80\left(\mathrm{~s}, 2 \mathrm{H}, \mathrm{CH}_{2}\right), 3.50(\mathrm{~s}, 6 \mathrm{H}$, $\left.\left(\mathrm{OCH}_{3}\right)_{2}\right) \cdot{ }^{13} \mathrm{C}$ NMR $\left(75 \mathrm{MHz}, \mathrm{CDCl}_{3}, \delta, \mathrm{ppm}\right): 150.4(\mathrm{C}), 146.7$ (C), $143.9(\mathrm{C}), 137.4(\mathrm{C}), 128.5(\mathrm{CH}), 127.7(\mathrm{CH}), 127.3(\mathrm{CH})$ $122.6(\mathrm{C}), 115.5(\mathrm{CH}), 102.3(\mathrm{CH}), 71.7\left(\mathrm{CH}_{2} \times 2\right), 71.0\left(\mathrm{CH}_{2} \times 2\right)$, $56.5\left(\mathrm{OCH}_{3} \times 2\right), 29.7\left(\mathrm{CH}_{2}\right)$. MS-EI $(\mathrm{m} / \mathrm{z}, \%): 652.2(100)$, $653.29\left(\mathrm{M}^{+}, 41\right)$

\section{Results and discussion}

The cross-coupling reaction to prepare the diphenyl methane analogues from readily available benzyl alcohol derivatives were studied with various halogenating agents such $\mathrm{SOCl}_{2}, \mathrm{PBr}_{3}$ and $\mathrm{MeSO}_{2} \mathrm{Cl}$. Initially, the benzyl alcohol was treated with thionyl chloride to produce the desired diphenyl methane. Unfortunately, the reaction proved to be unsuccessful and no desired product 6 was obtained. Further, the mono substituted benzyl alcohol derivatives $\mathbf{4}$ and $\mathbf{5}$, with electron denoting group (4'-OMe) and electron withdrawing group (4'$\mathrm{NO}_{2}$ ) were treated with thionyl chloride to see whether the electronic effect of substituents electronic affect the synthesis of corresponding diphenylmethane derivatives. However in both cases, only the corresponding halogenated products $\mathbf{7}$ and 8 were obtained (Table 1 ).

Further in this study, a tri-substituted brominated benzyl alcohol derivative $\mathbf{9}$ was then treated with thionyl chloride to get corresponding diphenylmethane derivative 10, which has close structural similarity with precursor of dibromo diphenyl methane derivative $\mathbf{2}$. We have observed that even in this case the reaction resulted in only the corresponding benzyl chloride product (Scheme 1). Though, we had obtained surprising results when we applied the above mentioned reaction conditions $\left(\mathrm{SOCl}_{2}, \mathrm{CH}_{2} \mathrm{Cl}_{2}\right)$ with another tri-substituted benzyl alcohol derivative $\mathbf{1 1}$ having all the substitutes of electron denoting nature. The cross coupling type reaction proceeded smoothly and region-selectively to give the desired diphenyl methane derivative $\mathbf{1 2}$ in excellent yield (83-85\%). The resultant compound $\mathbf{1 2}$ has a close structural similarity with dimethoxydip henylmethane derivative $\mathbf{2}$. This suggested that a highly substituted phenyl ring with electro denoting groups is necessarily required for such type of cross coupling reactions. The chemical method was further elaborated with other halogenating reagents such as $\mathrm{PBr}_{3}$ and $\mathrm{MeSO}_{2} \mathrm{Cl}$, which also afforded the same product 12 (Scheme 1).

A plausible mechanism of this reaction has also been layout in the following Scheme 2. The first step in mechanism showed the chlorination of the benzyl alcohol derivative $\mathbf{1 1}$ to corresponding benzyl chloride $\mathbf{1 3}$ which then tautomerize with its more stable carbocation specie $\mathbf{1 4}$ due to the effect of different electron denoting substituents on the phenyl ring.

Next the nucleophilic attack of the phenyl ring of benzyl alcohol $\mathbf{1 1}$ formed the diphenylmethane intermediate $\mathbf{1 5}$, which rapidly released a molecule of formaldehyde to retain the aromaticity. 

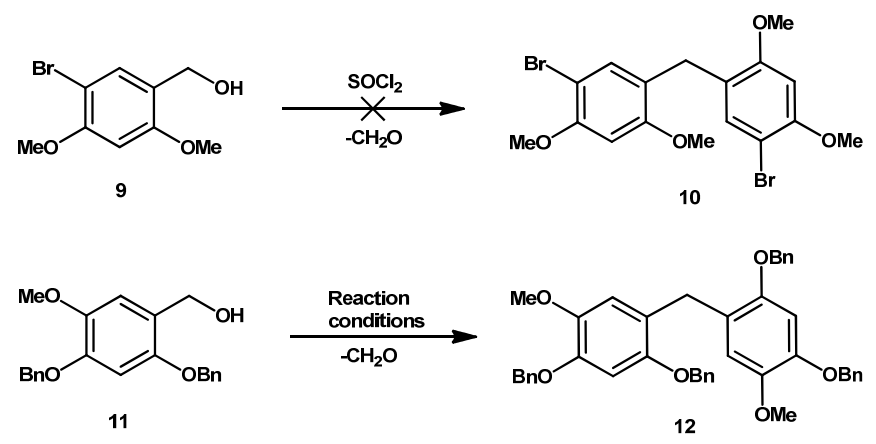

\begin{tabular}{lll}
\hline Entry & Reaction conditions & \% Yield \\
\hline 1 & $\mathrm{SOCl}_{2}, 0{ }^{\circ} \mathrm{C}, 45$ min. & 85 \\
2 & $\mathrm{PBr}_{3}, \mathrm{DCM}, 0{ }^{\circ} \mathrm{C}, 45$ min. & 83 \\
3 & $\mathrm{MeSO}_{2} \mathrm{Cl}, \mathrm{Et}_{3} \mathrm{~N}, \mathrm{DCM}, 0{ }^{\circ} \mathrm{C}$ to R.T. overnight & 83 \\
\hline
\end{tabular}

Scheme 1

The resultant coupling product $\mathbf{1 2}$ was obtained in excellent yield (83-85\%). The structure of the diphenyl methane analogue $\mathbf{1 2}$ was characterized with different analytical techniques which are including ${ }^{1} \mathrm{H}$ NMR, ${ }^{13} \mathrm{C}$ NMR spectroscopy and mass spectrometry. The structure was further confirmed by X-ray crystallography (Figure 2, Table 24).

Table 2. Crystal data and structure refinement for compound 12.

\begin{tabular}{|c|c|}
\hline Empirical formula & $\mathrm{C}_{43} \mathrm{H}_{40} \mathrm{O}_{6}$ \\
\hline Formula weight & 652.75 \\
\hline Temperature, $\mathrm{K}$ & $120(2)$ \\
\hline Crystal system & Monoclinic \\
\hline Wavelength, $\AA$ & 0.71073 \\
\hline Space group & $P 2_{1} / n$ \\
\hline a, $\AA$ & $15.7508(2)$ \\
\hline $\mathrm{b}, \AA$ & $9.7835(2)$ \\
\hline c, $\AA$ & $21.9581(4)$ \\
\hline$\alpha,{ }^{\circ}$ & 90.00 \\
\hline$\beta,{ }^{\circ}$ & $92.4920(10)$ \\
\hline$\gamma,^{\circ}$ & 90.00 \\
\hline Volume $/ \AA^{3}$ & $3380.50(10)$ \\
\hline $\mathrm{Z}$ & 4 \\
\hline$\rho_{\text {calc }}, \mathrm{mg} / \mathrm{mm}^{3}$ & 1.283 \\
\hline $\mathrm{m}, \mathrm{mm}^{-1}$ & 0.085 \\
\hline$F(000)$ & 1384.0 \\
\hline Crystal size, $\mathrm{mm}^{3}$ & $0.3 \times 0.03 \times 0.03$ \\
\hline Crystal & Needle, Colourless \\
\hline $2 \Theta$ range for data collection & 6.04 to $54.96^{\circ}$ \\
\hline Index ranges & $-20 \leq h \leq 20,-12 \leq k \leq 11,-28 \leq l \leq 28$ \\
\hline Reflections collected & 41608 \\
\hline Completeness to $\theta=27.48^{\circ}$ & $99.8 \%$ \\
\hline Absorption correction & Semi-empirical from equivalents \\
\hline Max. and min. transmission & 0.9975 and 0.9651 \\
\hline Refinement method & Full-matrix least-squares on $F^{2}$ \\
\hline Independent reflections & $7748[\mathrm{R}(\mathrm{int})=0.0720]$ \\
\hline Data/restraints/parameters & $7748 / 0 / 444$ \\
\hline Goodness-of-fit on $\mathrm{F}^{2}$ & 1.054 \\
\hline Final $R$ indexes $[I \geq 2 \sigma(I)]$ & $\mathrm{R}_{1}=0.0820, \mathrm{wR}_{2}=0.1560$ \\
\hline Final $\mathrm{R}$ indexes [all data] & $\mathrm{R}_{1}=0.1246, \mathrm{wR} 2=0.1796$ \\
\hline Largest diff. peak/hole, e $\AA^{-3}$ & $0.413 /-0.290$ \\
\hline
\end{tabular}

\section{Conclusion}

We have prepared a highly substituted diphenylmethane derivative 12, which has close structural similarity with the nature product $\mathbf{2}$, via a simple coupling reaction. The reaction with substituted benzyl alcohol $\mathbf{1 1}$ was optimized with three different types of halogenated reagents. The percentage yield of the desired diphenylmethane derivative $\mathbf{1 2}$ with each reaction condition was found excellent (83-85\%). Altogether, the chemical method is rapid and useful to excess the substituted diphenylmethane derivative $\mathbf{1 2}$ of biological importance and intermediates as well in the chemical industry.

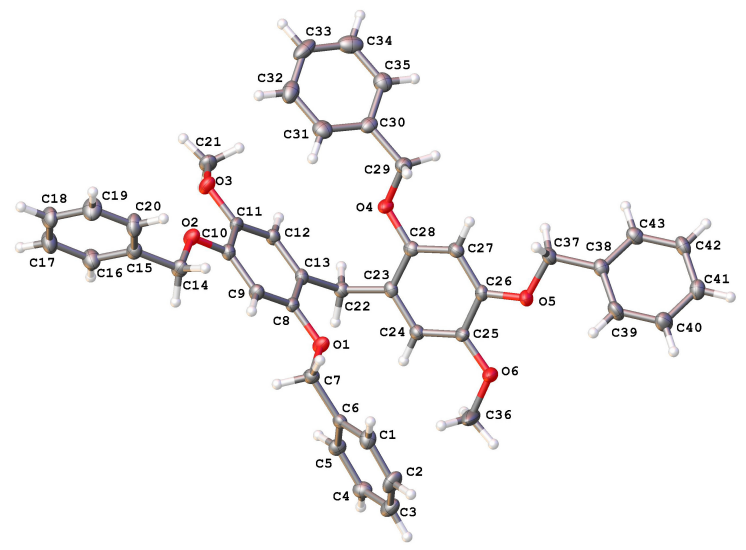

Figure 2. Crystal structure of compound 12.

Table 3. Bond lengths for compound 12.

\begin{tabular}{llll}
\hline Atom-Atom & Length, $\AA$ & Atom-Atom & Length, $\AA$ \\
\hline C1-C2 & $1.386(4)$ & C22-C23 & $1.522(4)$ \\
C1-C6 & $1.382(4)$ & C23-C24 & $1.394(4)$ \\
C2-C3 & $1.380(5)$ & C23-C28 & $1.388(4)$ \\
C3-C4 & $1.393(4)$ & C24-C25 & $1.388(4)$ \\
C4-C5 & $1.386(4)$ & C25-C26 & $1.400(4)$ \\
C5-C6 & $1.393(4)$ & C25-O6 & $1.373(3)$ \\
C6-C7 & $1.506(4)$ & C26-C27 & $1.388(4)$ \\
C7-01 & $1.429(3)$ & C26-05 & $1.368(3)$ \\
C8-C9 & $1.392(4)$ & C27-C28 & $1.395(4)$ \\
C8-C13 & $1.395(4)$ & C28-O4 & $1.384(3)$ \\
C8-01 & $1.381(3)$ & C29-C30 & $1.502(4)$ \\
C9-C10 & $1.392(4)$ & C29-O4 & $1.439(3)$ \\
C10-C11 & $1.400(4)$ & C30-C31 & $1.384(4)$ \\
C10-02 & $1.376(3)$ & C30-C35 & $1.387(4)$ \\
C11-C12 & $1.376(4)$ & C31-C32 & $1.384(5)$ \\
C11-03 & $1.382(3)$ & C32-C33 & $1.372(5)$ \\
C12-C13 & $1.391(4)$ & C33-C34 & $1.384(5)$ \\
C13-C22 & $1.515(4)$ & C34-C35 & $1.402(5)$ \\
C14-C15 & $1.503(4)$ & C36-O6 & $1.423(4)$ \\
C14-02 & $1.441(3)$ & C37-C38 & $1.500(4)$ \\
C15-C16 & $1.387(4)$ & C37-O5 & $1.437(3)$ \\
C15-C20 & $1.378(4)$ & C38-C39 & $1.395(4)$ \\
C16-C17 & $1.384(4)$ & C38-C43 & $1.388(4)$ \\
C17-C18 & $1.383(5)$ & C39-C40 & $1.387(4)$ \\
C18-C19 & $1.376(5)$ & C40-C41 & $1.388(4)$ \\
C19-C20 & $1.388(4)$ & C41-C42 & $1.386(4)$ \\
C21-03 & $1.425(3)$ & C42-C43 & $1.386(4)$ \\
\hline
\end{tabular}


<smiles>COc1cc(CO)c(OCCCCO)cc1OC</smiles>

11

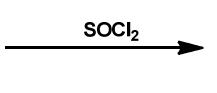<smiles>COc1cc(CCl)c(OC(=O)OCc2ccccc2)cc1OC</smiles>

13

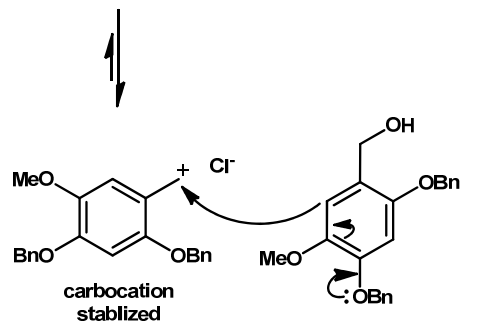

14

11

Scheme 2

Table 4. Bond angles for compound 12.

\begin{tabular}{llll}
\hline Atom-Atom-Atom & Angle, $^{\circ}$ & Atom-Atom-Atom & Angle, $^{\circ}$ \\
\hline C6-C1-C2 & $120.6(3)$ & C25-C24-C23 & $122.0(3)$ \\
C3-C2-C1 & $120.5(3)$ & C24-C25-C26 & $119.2(2)$ \\
C2-C3-C4 & $119.5(3)$ & O6-C25-C24 & $125.3(2)$ \\
C5-C4-C3 & $119.9(3)$ & O6-C25-C26 & $115.5(2)$ \\
C4-C5-C6 & $120.6(3)$ & C27-C26-C25 & $119.5(3)$ \\
C1-C6-C5 & $119.0(3)$ & O5-C26-C25 & $115.7(2)$ \\
C1-C6-C7 & $120.9(3)$ & O5-C26-C27 & $124.8(2)$ \\
C5-C6-C7 & $120.1(3)$ & C26-C27-C28 & $120.2(3)$ \\
O1-C7-C6 & $108.2(2)$ & C23-C28-C27 & $121.1(3)$ \\
C9-C8-C13 & $121.2(2)$ & O4-C28-C23 & $116.7(2)$ \\
O1-C8-C9 & $123.5(2)$ & O4-C28-C27 & $122.2(2)$ \\
O1-C8-C13 & $115.4(2)$ & O4-C29-C30 & $107.6(2)$ \\
C8-C9-C10 & $119.8(3)$ & C31-C30-C29 & $121.0(3)$ \\
C9-C10-C11 & $119.5(3)$ & C35-C30-C29 & $120.0(3)$ \\
O2-C10-C9 & $124.5(2)$ & C35-C30-C31 & $119.0(3)$ \\
O2-C10-C11 & $116.0(2)$ & C32-C31C30 & $120.8(3)$ \\
C12-C11-C10 & $119.5(2)$ & C33-C32-C31 & $120.3(3)$ \\
C12-C11-O3 & $124.7(2)$ & C32-C33-C34 & $120.0(3)$ \\
O3-C11-C10 & $115.7(2)$ & C33-C34-C35 & $119.8(3)$ \\
C11-C12-C13 & $122.1(3)$ & C30-C35-C34 & $120.1(3)$ \\
C8-C13-C22 & $121.2(2)$ & O5-C37-C38 & $107.4(2)$ \\
C12-C13-C8 & $117.8(2)$ & C39-C38-C37 & $121.0(2)$ \\
C12-C13-C22 & $121.1(2)$ & C43-C38-C37 & $120.1(3)$ \\
O2-C14-C15 & $108.5(2)$ & C43-C38-C39 & $118.9(3)$ \\
C16-C15-C14 & $120.1(3)$ & C40-C39-C38 & $120.3(3)$ \\
C20-C15-C14 & $121.3(3)$ & C41-C40-C39 & $120.4(3)$ \\
C20-C15-C16 & $118.6(3)$ & C42-C41-C40 & $119.5(3)$ \\
C17-C16-C15 & $120.6(3)$ & C41-C42-C43 & $120.2(3)$ \\
C18-C17-C16 & $120.2(3)$ & C38-C43-C42 & $120.8(3)$ \\
C19-C18-C17 & $119.5(3)$ & C8-01-C7 & $117.7(2)$ \\
C18-C19-C20 & $120.1(3)$ & C10-02-C14 & $116.2(2)$ \\
C15-C20-C19 & $121.0(3)$ & C11-O3-C21 & $116.2(2)$ \\
C13-C22-C23 & $115.2(2)$ & C28-O4-C29 & $116.5(2)$ \\
C24-C23-C22 & $119.5(2)$ & C26-O5-C37 & $118.2(2)$ \\
C28-C23-C22 & $122.6(2)$ & C25-O6-C36 & $116.2(2)$ \\
C28-C23-C24 & $117.9(2)$ & & \\
\hline & & & \\
\hline
\end{tabular}

\section{Acknowledgement}

We are thankful to Higher Education Commission of Pakistan for providing financial support of this project.

\section{Supplementary material}

CCDC-1001414 contains the supplementary crystallographic data for this paper. These data can be obtained free of charge via www.ccdc.cam.ac.uk/data request/cif, or by e-mailing data_request@ccdc.cam.ac.uk, or by contacting The Cambridge Crystallographic Data Centre, 12 Union Road, Cambridge CB2 1EZ, UK; fax: +44(0)1223-336033.

\section{References}

[1]. Bastock, T. W.; Clark, J. H. Speciality Chemicals, Elsevier, London, 1991.

[2]. Khadilkar, B. M.; Borkar, S. D. Chem. Technol. Biotechnol. 1998, 71, 209-212.

[3]. Commandeur, R.; Berger, N.; Jay, P.; Kervennal, J. J. Eur. Pat. Appl. EP 0442 986, 1991.

[4]. Feng, X. E.; Zhao, W. Y.; Ban, S. R.; Zhao, C. X.; Li, Q. S.; Lin, W. H. Int. J. Mol. Sci. 2011, 12, 6104-6115.

[5]. Balaydın, H. T.; Gılcin, I.; Menzek, A.; Goksu, S.; Sahin, E. J. Enzyme Inhib. Med. Chem. 2010, 25, 685-695.

[6]. Akutsu, M. A. C.; Otsuka, T. A. C. EP 1808223 B1, 2012.

[7]. Amano, S.; Tomita, H., US 5679730 A, 1997.

[8]. Sato, A.; Yida, Y.; Shimizi, I., DE-OS, 2210 133, 1972.

[9]. Stewart, M. I.; Carlson, O. K., US Patent, 3593 640, 1967.

[10]. Leus, A. M., US Patent, 3529 944, 1967.

[11]. Colon, M.; Guevara, P.; Gerwick, W. H.; Ballantine, D. J. Nat. Prod. 1987, 50, 368-374.

[12]. Wang, L.; Yin, Z. Q.; Shen, W. B.; Zhang, Q. W.; Ye, W. C. Helv. Chim. Acta 2007, 90, 1581-1585.

[13]. Ku, Y. Y.; Patel, R. R.; Sawick, D. P. Tetrahedron Lett. 1996, 37, 19491952.

[14]. Wei, Q.; Luo, Y.; Zhou, M.; Tao, F.; Zhang, G. Synth. Commun. 2005, 35 835-843.

[15]. Yin, D.; Li, C.; Tao, L.; Yu, N.; Hu, S.; Yin, D. J. Mol. Catal. A: Chem. 2006, 245, 260-265.

[16]. Olah, G. A. Friedel-Crafts Chemistry: Wiley, New York, 1973.

[17]. Duisenberg, A. J. M. J. Appl. Cryst. 1992, 25, 92-96.

[18]. Hooft, R. COLLECT. Nonius BV, Delft, The Netherlands, 1998.

[19]. Otwinowski, Z.; Minor, W. Methods in Enzymology; In Macromolecular Crystallography, Part A; Jr, C. W. C., Sweet, R. M., Eds.; Academic Press: New York, 1997; vol. 276.

[20]. Sheldrick, G. M. Acta Cryst. A 1990, 46, 467- 473.

[21]. Watkin, D. M.; Pearce, L.; Prout, C. K. Chemical Crystallography Laboratory; University of Oxford, 1993. 